\title{
A NEW REPRESENTATION ALGORITHM IN A FREE GROUP
}

\author{
Su-JeOng ChOI
}

\begin{abstract}
This paper presents a new representation algorithm which computes the representation for elements of a free group generated by two linear fractional transformations and also the justification of the algorithm in order to show how it operates correctly and efficiently according to inputs.
\end{abstract}

\section{Introduction}

Let $n \in \mathbb{N}$ with $n \geq 3$ and $\Gamma_{n}$ a free group [5] generated by two linear fractional transformations $A_{n}=\left[\begin{array}{ll}1 & n \\ 0 & 1\end{array}\right]$ and $B_{n}=\left[\begin{array}{ll}1 & 0 \\ n & 1\end{array}\right]$. Then every element of $\Gamma_{n}$ can be represented by a reduced word in $\left\{A_{n}, B_{n}\right\}^{ \pm}$, called the $X_{n}$-representation with $X_{n}=\left\{A_{n}, B_{n}\right\}$. Since $A_{n}$ and $B_{n}$ are the cases of $A_{1}{ }^{n}$ and $B_{1}{ }^{n}$, every element of $\Gamma_{n}$ can also be represented by a reduced word in $\left\{A_{1}, B_{1}\right\}^{ \pm}$, called the $X_{1}$-representation with $X_{1}=\left\{A_{1}, B_{1}\right\}$. Namely, the $X_{n}$-representation of each element of $\Gamma_{n}$ enables computation of the $X_{1}$ representation of the element. The $X_{1}$-representation of each element of $\Gamma_{n}$ is one of the following forms

$$
\begin{aligned}
& A_{1}{ }^{n u_{1}} B_{1}{ }^{n u_{2}} \cdots B_{1}{ }^{n u_{m-1}} A_{1}{ }^{n u_{m}}, \\
& A_{1}{ }^{n u_{1}} B_{1}{ }^{n u_{2}} \cdots A_{1}{ }^{n u_{m-1}} B_{1}{ }^{n u_{m}} \\
& B_{1}{ }^{n u_{1}} A_{1}{ }^{n u_{2}} \cdots A_{1}{ }^{n u_{m-1}} B_{1}{ }^{n u_{m}}, \\
& B_{1}{ }^{n u_{1}} A_{1}{ }^{n u_{2}} \cdots B_{1}{ }^{n u_{m-1}} A_{1}{ }^{n u_{m}},
\end{aligned}
$$

where $u_{i}$ is a nonzero integer and $m \in \mathbb{N}$.

In 2004, Grigoriev and Ponomarenko introduced the homomorphic publickey cryptosystem [4]. Then the secret key $n$ is hidden into the decryption scheme including the $X_{n}$-representation algorithm which outputs the $X_{n}$-repres entation of the ciphertext in the process of the decryption. Later on, this $X_{n^{-}}$ representation algorithm is modified to make it more clear and efficient in [2]. On the other hand, the attacker must strive to find the secret key $n$ of the

Received April 3, 2009.

2000 Mathematics Subject Classification. Primary 20C20, $20 \mathrm{~F} 05$.

Key words and phrases. linear fractional transformation, free group, representation, algorithm. 
cryptosystem. So the motivation of the design of the $X_{1}$-representation algorithm derives from cryptanalysis of the homomorphic public-key cryptosystem. Consequently the $X_{1}$-representation for elements of $\Gamma_{n}$ can be used to break the homomorphic public-key cryptosystem in [3].

In this paper, the $X_{1}$-representation algorithm is presented with the justification of the algorithm, which is stated explicitly with some properties of the two linear fractional transformations in order to show how it operates correctly and efficiently according to inputs. Note that the material of this paper is extracted from the PhD thesis of the author [1].

\section{Representation algorithm in a free group $\Gamma_{n}$}

In this section we introduce a new representation algorithm to compute the $X_{1}$-representation for elements of $\Gamma_{n}$ and also prove correctness of the algorithm with some properties of two linear fractional transformations $A_{n}{ }^{u}$ and $B_{n}{ }^{u}$ for a nonzero integer $u$. Let $D$ be a unit open disk in the complex plane $\mathbb{C}$ with the center 0, i.e., $D=\{z \in \mathbb{C}|| z \mid<1\}$ and a complement of the closure of $D$, $D^{c}=\mathbb{C}-\bar{D}=\{z \in \mathbb{C}|| z \mid>1\} .1_{X_{1}}$ denotes the empty word.

Assume that $n$ is unknown and $M \in \Gamma_{n}$. The following representation algorithm outputs the $X_{1}$-representation of $M$ either for $z=\frac{1}{2}$ or for $z=2$. Otherwise, it does the error message $\epsilon$. Once the algorithm first outputs the $X_{1}$-representation of $M$ for one of two $z$ values, it does not need to run for the other $z$ value as the goal is achieved.

\section{Algorithm}

Step 0

$w \leftarrow 1_{X_{1}}$

$L \leftarrow M$

Step 1

(1) $L(z)=0,|L(z)|=1, L(z)=\infty \Rightarrow$ output $\epsilon$.

(2) $|L(z)|>1 \Rightarrow$ compute $e, \mu$ s.t. $L(z)=e+\mu, e \in \mathbb{Z},-\frac{1}{2}<\mu \leq \frac{1}{2}$ and go to Step 2 .

(3) $|L(z)|<1 \Rightarrow$ compute $e, \mu$ s.t. $\frac{1}{L(z)}=e+\mu, e \in \mathbb{Z},-\frac{1}{2}<\mu \leq \frac{1}{2}$ and go to Step 3 .

Step 2

(1) $C \leftarrow A_{1}{ }^{e}$ and $w \leftarrow w C$.

(2) $C=I \Rightarrow$ output $\epsilon$.

(3) $L \leftarrow C^{-1} L$

(4) $L=I \Rightarrow$ output $w$. Otherwise, return Step 1 .

Step 3

(1) $C \leftarrow B_{1}{ }^{e}$ and $w \leftarrow w C$.

(2) $C=I \Rightarrow$ output $\epsilon$.

(3) $L \leftarrow C^{-1} L$

(4) $L=I \Rightarrow$ output $w$. Otherwise, return Step 1 .

Next, some properties of the two linear fractional transformations are as follows. 
Lemma 2.1 ([2]). For $z \in D, A_{n}{ }^{u}(z) \in D^{c}$.

Lemma $2.2([2])$. For $z \in D^{c}, B_{n}{ }^{u}(z) \in D$.

Theorem 2.3 ([2]). The following properties hold:

(1) $A_{n}{ }^{u_{1}} B_{n}{ }^{u_{2}} \cdots B_{n}{ }^{u_{m-1}} A_{n}{ }^{u_{m}} \in D^{c}$ for $z \in D$.

(2) $A_{n}{ }^{u_{1}} B_{n}{ }^{u_{2}} \cdots A_{n}{ }^{u_{m-1}} B_{n}{ }^{u_{m}} \in D^{c}$ for $z \in D^{c}$.

(3) $B_{n}{ }^{u_{1}} A_{n}{ }^{u_{2}} \cdots A_{n}{ }^{u_{m-1}} B_{n}{ }^{u_{m}} \in D$ for $z \in D^{c}$.

(4) $B_{n}{ }^{u_{1}} A_{n}{ }^{u_{2}} \cdots B_{n}{ }^{u_{m-1}} A_{n}{ }^{u_{m}} \in D$ for $z \in D$.

Theorem 2.4. Let $n \geq 3$ and $z \in \mathbb{R}$ such that $|z|<\frac{1}{2}$. Then $\left|A_{n}{ }^{u}(z)\right|>\frac{5}{2}$.

Proof. For $n \geq 3$ and $z \in \mathbb{R}$ s.t. $|z|<\frac{1}{2}$, by Lemma $2.1 A_{n}^{u}(z)=n u+z \in D^{c}$. If $u \geq 1$, then $\frac{5}{2} \leq n u-\frac{1}{2}<n u+z<n u+\frac{1}{2}$ and so $A_{n}{ }^{u}(z)>\frac{5}{2}$. If $u<-1$, then $n u-\frac{1}{2}<n u+z<n u+\frac{1}{2}<-\frac{5}{2}$ and so $A_{n}{ }^{u}(z)<-\frac{5}{2}$.

Theorem 2.5. For $n \geq 3$ and $z \in \mathbb{R} \cap D^{c},\left|B_{n}{ }^{u}(z)\right|<\frac{1}{2}$.

Proof. Let $n \geq 3$ and $z \in \mathbb{R} \cap D^{c}$. If $u \geq 1$, then $0<\frac{1}{n u+\frac{1}{z}}<\frac{1}{n u-1} \leq \frac{1}{2}$ and so $0<B_{n}{ }^{u}(z)<\frac{1}{2}$. If $u<-1$, then $-\frac{1}{2}<\frac{1}{n u+1}<\frac{1}{n u+\frac{1}{z}}<0$ and so $-\frac{1}{2}<B_{n}{ }^{u}(z)<0$.

It should be noticed that both properties above are not guaranteed for $n \geq 2$. In other words, let $n \geq 2$ and $z \in \mathbb{R} \cap D^{c}$, then $\left|B_{n}{ }^{u}(z)\right|<1$, but for $n \geq 3$, $\left|B_{n}{ }^{u}(z)\right|<\frac{1}{2}$. Now the justification of the algorithm is being carried out with some characteristics of the two linear fractional transformations. So it shows how the algorithm works correctly and efficiently according to inputs. For the sake of avoiding the similarity of proofs, one of the $X_{1}$-representation forms is taken for the verification of the algorithm.

Theorem 2.6. If a matrix $M=A_{n}{ }^{u_{1}} B_{n}{ }^{u_{2}} \cdots A_{n}{ }^{u_{m-1}} B_{n}{ }^{u_{m}}$ is input to the algorithm $\left(z=\frac{1}{2}\right)$ with even $m \geq 2$, then it outputs $\epsilon$ as the error message.

Proof. Let

$$
M=A_{n}{ }^{u_{1}} B_{n}{ }^{u_{2}} \cdots A_{n}{ }^{u_{m-1}} B_{n}{ }^{u_{m}} \in \Gamma_{n} \text { and } \beta_{1}=B_{n}{ }^{u_{2}} \cdots A_{n}{ }^{u_{m-1}} B_{n}{ }^{u_{m}}\left(\frac{1}{2}\right) .
$$

Then $L\left(\frac{1}{2}\right)=A_{n}{ }^{u_{1}} B_{n}{ }^{u_{2}} \cdots A_{n}{ }^{u_{m-1}} B_{n}{ }^{u_{m}}\left(\frac{1}{2}\right)=n u_{1}+\beta_{1}$.

Case 1 : If $n=3$ and $u_{m}=-1$, then $\left|B_{n}{ }^{u_{m}}\left(\frac{1}{2}\right)\right|=1$ and in Step 1 of the first iteration, by Theorem $2.3(2)$,

$$
L\left(\frac{1}{2}\right)=A_{n}{ }^{u_{1}} B_{n}{ }^{u_{2}} \cdots B_{n}{ }^{u_{m-2}}\left(n u_{m-1}-1\right) \in D^{c} .
$$

As $A_{n}{ }^{u_{m-1}} B_{n}{ }^{u_{m}}\left(\frac{1}{2}\right)=A_{n}^{u_{m-1}}(-1) \in D^{c}$, by Theorem $2.5,\left|\beta_{1}\right|<\frac{1}{2}$, so that $e=n u_{1}$ and $\mu=\beta_{1}$.

Case 2: If $n \neq 3$ or $u_{m} \neq-1$, then $\left|B_{n}{ }^{u_{m}}\left(\frac{1}{2}\right)\right|<1$. By Theorem 2.3(1),

$$
L\left(\frac{1}{2}\right)=A_{n}^{u_{1}} B_{n}^{u_{2}} \cdots A_{n}^{u_{m-1}}\left(\frac{1}{n u_{m}+2}\right) \in D^{c}
$$


and by Theorem 2.5, $\left|\beta_{1}\right|<\frac{1}{2}$, so that $e=n u_{1}$ and $\mu=\beta_{1}$. In Step 2 of the first iteration of those cases, $C=A_{1}{ }^{e}=A_{1}{ }^{n u_{1}}, w=w C=A_{1}{ }^{n u_{1}}$ and $L=C^{-1} L=B_{n}{ }^{u_{2}} \cdots A_{n}{ }^{u_{m-1}} B_{n}{ }^{u_{m}} \neq I$. So return Step 1 .

Suppose that for $1 \leq i-1<m-2$, in Step 2 of the $i-1$ th iteration, $L=C^{-1} L=B_{n}{ }^{u_{i}} A_{n}{ }^{u_{i+1}} \cdots A_{n}{ }^{u_{m-1}} B_{n}{ }^{u_{m}}$ or in Step 3 of the $i-1$ th iteration $L=C^{-1} L=A_{n}{ }^{u_{i}} B_{n}{ }^{u_{i+1}} \cdots A_{n}{ }^{u_{m-1}} B_{n}{ }^{u_{m}}$ according as $i-1$ is odd or even.

For even $i$, let $L=B_{n}{ }^{u_{i}} A_{n}{ }^{u_{i+1}} \cdots A_{n}{ }^{u_{m-1}} B_{n}{ }^{u_{m}}$ in Step 1 of the $i$ th iteration and $\alpha_{i}=A_{n}{ }^{u_{i+1}} B_{n}{ }^{u_{i+2}} \cdots A_{n}{ }^{u_{m-1}} B_{n}{ }^{u_{m}}\left(\frac{1}{2}\right)$. Then

$$
L\left(\frac{1}{2}\right)=B_{n}^{u_{i}}\left(\alpha_{i}\right)=\frac{1}{n u_{i}+\frac{1}{\alpha_{i}}} \text {. }
$$

Case 1 : If $n=3$ and $u_{m}=-1$, then $\left|B_{n}{ }^{u_{m}}\left(\frac{1}{2}\right)\right|=1$ and by Theorem 2.3(3),

$$
L\left(\frac{1}{2}\right)=B_{n}{ }^{u_{i}} A_{n}{ }^{u_{i+1}} \cdots B_{n}{ }^{u_{m-2}}\left(n u_{m-1}-1\right) \in D .
$$

As $A_{n}{ }^{u_{m-1}} B_{n}{ }^{u_{m}}\left(\frac{1}{2}\right)=A_{n}{ }^{u_{m-1}}(-1) \in D^{c}$, by Theorem 2.5,

$$
\left|B_{n}{ }^{u_{i+2}} \cdots A_{n}{ }^{u_{m-1}} B_{n}{ }^{u_{m}}\left(\frac{1}{2}\right)\right|<\frac{1}{2} \text {. }
$$

By Theorem 2.4, $\frac{1}{\left|\alpha_{i}\right|}<\frac{2}{5}$ and as $\frac{1}{L\left(\frac{1}{2}\right)}=n u_{i}+\frac{1}{\alpha_{i}}$,

$$
e=n u_{i} \text { and } \mu=\frac{1}{\alpha_{i}} \text {. }
$$

Case 2 : If $n \neq 3$ or $u_{m} \neq-1$, then $\left|B_{n}{ }^{u_{m}}\left(\frac{1}{2}\right)\right|<1$ and by Theorem 2.3(4),

$$
L\left(\frac{1}{2}\right)=B_{n}{ }^{u_{i}} A_{n}{ }^{u_{i+1}} \cdots A_{n}{ }^{u_{m-1}}\left(\frac{1}{n u_{m}+2}\right) \in D .
$$

By Lemma 2.1, $A_{n}{ }^{u_{m-1}}\left(\frac{1}{n u_{m}+2}\right) \in D^{c}$ and by Theorem 2.5,

$$
\left|B_{n}{ }^{u_{i+2}} \cdots A_{n}^{u_{m-1}} B_{n}^{u_{m}}\left(\frac{1}{2}\right)\right|<\frac{1}{2} .
$$

By Theorem 2.4, $\frac{1}{\left|\alpha_{i}\right|}<\frac{2}{5}$ and $\frac{1}{L\left(\frac{1}{2}\right)}=n u_{i}+\frac{1}{\alpha_{i}}$, so that $e=n u_{i}$ and $\mu=\frac{1}{\alpha_{i}}$. In Step 3 of the $i$ th iteration of those cases, $C=B_{1}{ }^{e}=B_{1}{ }^{n u_{i}}, w=w C=$ $A_{1}{ }^{n u_{1}} B_{1}{ }^{n u_{2}} \cdots A_{1}{ }^{n u_{i-1}} B_{1}{ }^{n u_{i}}$ and $L=C^{-1} L=A_{n}{ }^{u_{i+1}} \cdots A_{n}{ }^{u_{m-1}} B_{n}{ }^{u_{m}} \neq I$. So return Step 1 .

For odd $i$, let $L=A_{n}{ }^{u_{i}} B_{n}{ }^{u_{i+1}} \cdots A_{n}{ }^{u_{m-1}} B_{n}{ }^{u_{m}}$ and $\beta_{i}=B_{n}{ }^{u_{i+1}} \cdots A_{n}{ }^{u_{m-1}}$ $B_{n}{ }^{u_{m}}\left(\frac{1}{2}\right)$. Then $L\left(\frac{1}{2}\right)=n u_{i}+\beta_{i}$.

Case 1: If $n=3$ and $u_{m}=-1$, then $\left|B_{n}{ }^{u_{m}}\left(\frac{1}{2}\right)\right|=1$ and by Theorem 2.3(2),

$$
L\left(\frac{1}{2}\right)=A_{n}{ }^{u_{i}} B_{n}{ }^{u_{i+1}} \cdots B_{n}{ }^{u_{m-2}}\left(n u_{m-1}-1\right) \in D^{c} .
$$

As $A_{n}{ }^{u_{m-1}} B_{n}{ }^{u_{m}}\left(\frac{1}{2}\right)=A_{n}{ }^{u_{m-1}}(-1) \in D^{c}$, by Theorem $2.5,\left|\beta_{i}\right|<\frac{1}{2}$. Hence

$$
e=n u_{i} \text { and } \mu=\beta_{i} \text {. }
$$

Case 2 : If $n \neq 3$ or $u_{m} \neq-1$, then $\left|B_{n}{ }^{u_{m}}\left(\frac{1}{2}\right)\right|<1$. By Theorem 2.3(1),

$$
L\left(\frac{1}{2}\right)=A_{n}{ }^{u_{i}} B_{n}{ }^{u_{i+1}} \cdots A_{n}{ }^{u_{m-1}}\left(\frac{1}{n u_{m}+2}\right) \in D^{c}
$$


and by Theorem 2.5, $\left|\beta_{i}\right|<\frac{1}{2}$, so that $e=n u_{i}$ and $\mu=\beta_{i}$. In Step 2 of the $i$ th iteration of those cases,

$$
\begin{gathered}
C=A_{1}{ }^{e}=A_{1}{ }^{n u_{i}}, w=w C=A_{1}{ }^{n u_{1}} B_{1}{ }^{n u_{2}} \cdots B_{1}{ }^{u_{i-1}} A_{1}{ }^{n u_{i}} \\
\text { and } L=C^{-1} L=B_{n}{ }^{u_{i+1}} \cdots A_{n}{ }^{u_{m-1}} B_{n}{ }^{u_{m}} \neq I .
\end{gathered}
$$

So return Step 1 .

For $i=m-1$, the algorithm runs with $L=C^{-1} L=A_{n}{ }^{u_{m-1}} B_{n}{ }^{u_{m}}$ in Step 3 of the $m-2$ th iteration.

Case 1 : If $n=3$ and $u_{m}=-1$, then $\left|B_{n}{ }^{u_{m}}\left(\frac{1}{2}\right)\right|=1$ and

$$
L\left(\frac{1}{2}\right)=A_{n}{ }^{u_{m-1}} B_{n}{ }^{u_{m}}\left(\frac{1}{2}\right)=A_{n}{ }^{u_{m-1}}(-1) \in D^{c} .
$$

In Step 1 of the $m-1$ th iteration,

$$
e=n u_{m-1}-1 \text { and } \mu=0 .
$$

In Step 2 of the $m-1$ th iteration,

$$
\begin{gathered}
C=A_{1}^{e}=A_{1}^{n u_{m-1}-1}, \\
w=w C=A_{1}{ }^{n u_{1}} B_{1}{ }^{n u_{2}} \cdots B_{1}{ }^{n u_{m-2}} A_{1}{ }^{n u_{m-1}-1}
\end{gathered}
$$

and

$$
L=C^{-1} L=A_{1} B_{n}{ }^{u_{m}} \neq I .
$$

So return Step 1 .

For $i=m$ of case 1 , the algorithm runs with $L=A_{1} B_{n}{ }^{u_{m}}$ in Step 1 of the $m$ th iteration and then

$$
L\left(\frac{1}{2}\right)=A_{1} B_{n}^{u_{m}}\left(\frac{1}{2}\right)=A_{1}(-1)=0 .
$$

Therefore the algorithm outputs $\epsilon$ as the error message and then it terminates. Case 2 : If $n \neq 3$ or $u_{m} \neq-1$, then $\left|B_{n}{ }^{u_{m}}\left(\frac{1}{2}\right)\right|<1$ and

$$
L\left(\frac{1}{2}\right)=A_{n}^{u_{m-1}} B_{n}^{u_{m}}\left(\frac{1}{2}\right)=A_{n}^{u_{m-1}}\left(\frac{1}{n u_{m}+2}\right) \in D^{c} .
$$

Since $\left|B_{n}{ }^{u_{m}}\left(\frac{1}{2}\right)\right| \leq \frac{1}{2}$,

$$
e=n u_{m-1} \text { and } \mu=\frac{1}{n u_{m}+2} .
$$

In Step 2 of the $m-1$ th iteration,

$$
\begin{gathered}
C=A_{1}{ }^{e}=A_{1}{ }^{n u_{m-1}}, \\
w=w C=A_{1}{ }^{n u_{1}} B_{1}{ }^{n u_{2}} \cdots B_{1}{ }^{n u_{m-2}} A_{1}{ }^{n u_{m-1}} \\
\text { and } L=C^{-1} L=B_{n}{ }^{u_{m}} \neq I .
\end{gathered}
$$

So return Step 1 .

For $i=m$ of case 2 , the algorithm runs with $L=B_{n}{ }^{u_{m}}$ in Step 1 of the $m$ th iteration.

Case 1 : If $n=3$ and $u=-1$, then in Step 1 of the $m$ th iteration, $\left|L\left(\frac{1}{2}\right)\right|=1$. So the algorithm outputs $\epsilon$ as the error message and then it terminates. 
Case 2 : If $n \neq 3$ or $u_{m} \neq-1$, then in Step 1 of the $m$ th iteration, $\left|L\left(\frac{1}{2}\right)\right| \leq \frac{1}{2}$ and so

$$
e=\frac{1}{L\left(\frac{1}{2}\right)}=n u_{m}+2 \text { and } \mu=0 .
$$

In Step 3 of the $m$ th iteration,

$$
\begin{gathered}
C=B_{1}{ }^{e}=B_{1}{ }^{n u_{m}+2} \\
w=w C=A_{1}{ }^{n u_{1}} B_{1}{ }^{n u_{2}} \cdots B_{1}{ }^{n u_{m-2}} A_{1}{ }^{n u_{m-1}} B_{1}{ }^{n u_{m}+2}
\end{gathered}
$$

and

$$
L=C^{-1} L=B_{1}^{-n u_{m}-2} B_{n}^{u_{m}}=B_{1}^{-2} \neq I .
$$

So return Step 1.

For $i=m+1$ of case 2 , the algorithm runs with $L=C^{-1} L=B_{1}^{-2}$ in Step 3 of the $m$ th iteration and then

$$
L\left(\frac{1}{2}\right)=B_{1}^{-2}\left(\frac{1}{2}\right)=\infty
$$

in Step 1 of the $m+1$ th iteration. Therefore the algorithm outputs $\epsilon$ as the error message and then it terminates.

Theorem 2.7. If a matrix $M=A_{n}{ }^{u_{1}} B_{n}{ }^{u_{2}} \cdots A_{n}{ }^{u_{m-1}} B_{n}{ }^{u_{m}}$ is input to the algorithm $(z=2)$ with even $m \geq 2$, then it outputs $A_{1}{ }^{n u_{1}} B_{1}{ }^{n u_{2}} \cdots A_{1}{ }^{n u_{m-1}} B_{1}{ }^{n u_{m}}$ as the $X_{1}$-representation of $M$.

Proof. Let

$$
M=A_{n}{ }^{u_{1}} B_{n}{ }^{u_{2}} \cdots A_{n}{ }^{u_{m-1}} B_{n}{ }^{u_{m}} \in \Gamma_{n} \text { and } \beta_{1}=B_{n}{ }^{u_{2}} \cdots A_{n}{ }^{u_{m-1}} B_{n}{ }^{u_{m}}(2) .
$$

Then

$$
L(2)=A_{n}{ }^{u_{1}} B_{n}{ }^{u_{2}} \cdots A_{n}{ }^{u_{m-1}} B_{n}{ }^{u_{m}}(2)=n u_{1}+\beta_{1} .
$$

By Theorem 2.3(2), $L(2) \in D^{c}$ and by Theorem 2.5, $\left|\beta_{1}\right|<\frac{1}{2}$, so that $e=n u_{1}$ and $\mu=\beta_{1}$. In Step 2 of the first iteration,

$$
\begin{aligned}
C & =A_{1}{ }^{e}=A_{1}{ }^{n u_{1}}, w=w C=A_{1}{ }^{n u_{1}} \\
\text { and } L & =C^{-1} L=B_{n}{ }^{u_{2}} \cdots A_{n}{ }^{u_{m-1}} B_{n}{ }^{u_{m}} \neq I .
\end{aligned}
$$

So return Step 1.

Assume that for $1 \leq i-1<m-1$, in Step 3 of the $i-1$ th iteration $L=C^{-1} L=A_{n}{ }^{u_{i}} B_{n}{ }^{u_{i+1}} \cdots A_{n}{ }^{u_{m-1}} B_{n}{ }^{u_{m}}$ or in Step 2 of the $i-1$ th iteration $L=C^{-1} L=B_{n}{ }^{u_{i}} A_{n}{ }^{u_{i+1}} \cdots A_{n}{ }^{u_{m-1}} B_{n}{ }^{u_{m}}$ according as $i-1$ is even or odd.

For even $i$, in Step 1 of the $i$ th iteration, let $L=B_{n}{ }^{u_{i}} A_{n}{ }^{u_{i+1}} \cdots A_{n}{ }^{u_{m-1}} B_{n}{ }^{u_{m}}$ $\in \Gamma_{n}$ and $\alpha_{i}=A_{n}{ }^{u_{i+1}} \cdots A_{n}{ }^{u_{m-1}} B_{n}{ }^{u_{m}}(2)$. Then

$$
L(2)=B_{n}{ }^{u_{i}}\left(\alpha_{i}\right)=\frac{1}{n u_{i}+\frac{1}{\alpha_{i}}} .
$$

By Theorem 2.5, $|L(2)|<\frac{1}{2}$ and by Theorem $2.4, \frac{1}{\left|\alpha_{i}\right|}<\frac{2}{5}$, so that 


$$
e=n u_{i} \text { and } \mu=\frac{1}{\alpha_{i}} \text {. }
$$

In Step 3 of the $i$ th iteration, $C=B_{1}{ }^{e}=B_{1}{ }^{n u_{i}}, w=w C=A_{1}{ }^{n u_{1}} B_{1}{ }^{n u_{2}} \ldots$ $A_{1}{ }^{n u_{i-1}} B_{1}{ }^{n u_{i}}$ and $L=C^{-1} L=A_{n}{ }^{u_{i+1}} \cdots A_{n}{ }^{u_{m-1}} B_{n}{ }^{u_{m}} \neq I$. So return Step 1 .

For odd $i$, let $L=A_{n}{ }^{u_{i}} B_{n}{ }^{u_{i+1}} \cdots A_{n}{ }^{u_{m-1}} B_{n}{ }^{u_{m}} \in \Gamma_{n}$ in Step 1 of the $i$ th iteration and $\beta_{i}=B_{n}{ }^{u_{i+1}} \cdots A_{n}{ }^{u_{m-1}} B_{n}{ }^{u_{m}}(2)$. Then

$$
L(2)=A_{n}{ }^{u_{i}} B_{n}{ }^{u_{i+1}} \cdots A_{n}{ }^{u_{m-1}} B_{n}{ }^{u_{m}}=n u_{i}+\beta_{i}
$$

and by Theorem 2.5, $\left|\beta_{i}\right|<\frac{1}{2}$, so that $e=n u_{i}$ and $\mu=\beta_{i}$. In Step 2 of the $i$ th iteration, $C=A_{1}{ }^{e}=A_{1}{ }^{n u_{i}}, w=w C=A_{1}{ }^{n u_{1}} B_{1}{ }^{n u_{2}} \cdots B_{1}{ }^{n u_{i-1}} A_{1}{ }^{n u_{i}}$ and $L=C^{-1} L=B_{n}{ }^{u_{i+1}} \cdots A_{n}{ }^{u_{m-1}} B_{n}{ }^{u_{m}} \neq I$. So return Step 1 .

If $i=m$, then the algorithm runs with $L=B_{n}{ }^{u_{m}}$ in Step 1 of the $m$ th iteration and by Theorem 2.5, $|L(2)|<\frac{1}{2}$. As $\frac{1}{L(2)}=n u_{m}+\frac{1}{2}$,

$$
e=n u_{m} \text { and } \mu=\frac{1}{2} \text {. }
$$

In Step 3 of the $m$ th iteration,

$$
C=B_{1}^{e}=B_{1}^{n u_{m}}, w=w C=A_{1}^{n u_{1}} B_{1}{ }^{n u_{2}} \cdots A_{1}{ }^{n u_{m-1}} B_{1}{ }^{n u_{m}}
$$

and

$$
L=C^{-1} L=B_{1}^{-n u_{m}} B_{n}^{u_{m}}=I .
$$

Hence the algorithm outputs

$$
A_{1}^{n u_{1}} B_{1}^{n u_{2}} \cdots A_{1}^{n u_{m-1}} B_{1}^{n u_{m}}
$$

as the $X_{1}$-representation of $M$ and then it terminates.

\section{Theorem 2.8.}

(1) If a matrix $M=A_{n}{ }^{u_{1}} B_{n}{ }^{u_{2}} \cdots B_{n}{ }^{u_{m-1}} A_{n}{ }^{u_{m}}$ is input to the algorithm $\left(z=\frac{1}{2}\right)$ with odd $m \geq 3$, then it outputs $A_{1}{ }^{n u_{1}} B_{1}{ }^{n u_{2}} \cdots B_{1}^{n u_{m-1}} A_{1}^{n u_{m}}$ as the $X_{1}$-representation of $M$.

(2) If a matrix $M=A_{n}{ }^{u_{1}} B_{n}{ }^{u_{2}} \cdots B_{n}{ }^{u_{m-1}} A_{n}{ }^{u_{m}}$ is input to the algorithm $(z=2)$ with odd $m \geq 3$, then it outputs $\epsilon$ as the error message.

(3) If a matrix $M=B_{n}{ }^{u_{1}} A_{n}{ }^{u_{2}} \cdots B_{n}{ }^{u_{m-1}} A_{n}{ }^{u_{m}}$ is input to the algorithm $\left(z=\frac{1}{2}\right)$ with even $m \geq 2$, then it outputs $B_{1}{ }^{n u_{1}} A_{1}{ }^{n u_{2}} \cdots B_{1}{ }^{n u_{m-1}} A_{1}{ }^{n u_{m}}$ as the $X_{1}$-representation of $M$.

(4) If a matrix $M=B_{n}{ }^{u_{1}} A_{n}{ }^{u_{2}} \cdots B_{n}{ }^{u_{m-1}} A_{n}{ }^{u_{m}}$ is input to the algorithm $(z=2)$ with even $m \geq 2$, then it outputs $\epsilon$ as the error message.

(5) If a matrix $M=B_{n}{ }^{{ }_{1}} A_{n}{ }^{u_{2}} \cdots A_{n}{ }^{u_{m-1}} B_{n}{ }^{u_{m}}$ is input to the algorithm $\left(z=\frac{1}{2}\right)$ with odd $m \geq 3$, then it outputs $\epsilon$ as the error message.

(6) If a matrix $M=B_{n}{ }^{u_{1}} A_{n}{ }^{u_{2}} \cdots A_{n}{ }^{u_{m-1}} B_{n}{ }^{u_{m}}$ is input to the algorithm $(z=2)$ with odd $m \geq 3$, then it outputs $B_{1}{ }^{n u_{1}} A_{1}{ }^{n u_{2}} \cdots A_{1}{ }^{n u_{m-1}} B_{1}{ }^{n u_{m}}$ as the $X_{1}$-representation of $M$.

Proof. It is similar to the proofs of Theorem 2.6 and Theorem 2.7. 


\section{Conclusions}

The purpose of this paper is to design a new representation algorithm for elements of a free group generated by the two linear fractional transformations and also show proofs of correctness of the algorithm, which are dominant in this note. This work seemingly looks more or less straightforward, but indeed, it clarifies even subtle cases in which the algorithm may not work properly. Subsequently the algorithm comes to have computational efficiency. Moreover some theoretical background of the algorithm is apparently shown with the properties of the two linear fractional transformations. Further from combinatorial group theoretical point of view, the $X_{1}$-representation algorithm might give an insight to design algorithms for other groups such as symplectic group $\operatorname{Sp}(2,1)$, special linear group $\operatorname{SL}(2, \mathbb{Z})$ or general linear group $\mathrm{GL}(2, \mathbb{Z})$. In practice programming of the algorithm and demonstrations with experiments appear in [1].

\section{References}

[1] S. J. Choi, Cryptanalysis of a homomorphic public-key cryptosystem over a finite group, Ph.D. thesis, University of London, 2006.

[2] _ Representation algorithms in some free groups, J. Korea Soc. Math. Educ. Ser. B : Pure Appl. Math. 15 (2008), no. 3, 229-243.

[3] S. J. Choi, S. R. Blackburn, and P. R. Wild, Cryptanalysis of a homomorphic public-key cryptosystem over a finite group, J. Math. Crypt. 1 (2007), no. 4, 351-358.

[4] D. Grigoriev and I. Ponomarenko, Homomorphic public key cryptosystems over groups and rings, Complexity of computations and proofs, Quad. Math. 13 (2004), 305-325.

[5] R. C. Lyndon and P. E. Schupp, Combinatorial Group Theory, Springer, Berlin, New York, 1977.

Department of Mathematics

DONG-A University

BUSAN 604-714, Korea

E-mail address: sjchoi09@donga.ac.kr 